\title{
Exosomes and tumor-mediated immune suppression
}

\author{
Theresa L. Whiteside \\ Departments of Pathology, Immunology, and Otolaryngology, University of Pittsburgh School of Medicine and University of Pittsburgh Cancer Institute, Pittsburgh, Pennsylvania, USA.
}

\begin{abstract}
Tumor-derived exosomes (TEX) are harbingers of tumor-induced immune suppression: they carry immunosuppressive molecules and factors known to interfere with immune cell functions. By delivering suppressive cargos consisting of proteins similar to those in parent tumor cells to immune cells, TEX directly or indirectly influence the development, maturation, and antitumor activities of immune cells. TEX also deliver genomic DNA, mRNA, and microRNAs to immune cells, thereby reprogramming functions of responder cells to promote tumor progression. TEX carrying tumor-associated antigens can interfere with antitumor immunotherapies. TEX also have the potential to serve as noninvasive biomarkers of tumor progression. In the tumor microenvironment, TEX may be involved in operating numerous signaling pathways responsible for the downregulation of antitumor immunity.
\end{abstract}

\section{Introduction}

Exosomes, small membrane-bound vesicles, are a class of extracellular vesicles (EVs) made and released by most, if not all, cells. They are present in all body fluids (1-4) and have recently been in the limelight because of their potential role as communication vehicles between cells and as a new, noninvasive type of cancer biomarker (5-7). My first encounter with tumor-derived exosomes (TEX) occurred in the early 2000s, when an observation that sera of cancer patients induced DNA fragmentation in human activated primary $\mathrm{T}$ cells attracted my attention. Sera of healthy donors did not induce apoptosis of activated $\mathrm{CD} 8^{+} \mathrm{T}$ cells (8). Upon ultracentrifugation of cancer patients' sera, it turned out that the pelleted vesicular material contained apoptosis-inducing factors. Later, it became clear that small vesicles sized at approximately $100 \mathrm{~nm}$ (i.e., virus size) and carrying FasL were responsible for apoptosis of activated, FAS-expressing $\mathrm{T}$ cells (8). Studies of this phenomenon using cultured tumor cells showed that these vesicles were produced in abundance and induced a variety of functional alterations in immune cells.

Exosome secretion by cells seems to be a physiological phenomenon that occurs spontaneously. In fact, in the early 1980s, exosome secretion was thought to be necessary to remove cellular waste (9). On the basis of studies of exosome content and their interactions with recipient cells, exosomes are now thought to mediate "targeted" information transfer (10). TEX carry a cargo of molecules that is different from that of exosomes made by normal cells and, consequently, TEX mediate distinct biological effects (11). This Review will consider TEX, their cargo, and biological functions in the context of tumor-mediated immune suppression, which accompanies tumor growth and facilitates tumor escape from the host immune system (12).

\section{Morphological and molecular features of TEX}

TEX are the smallest type of EVs. EV nomenclature is confusing, because EVs encompass a wide variety of poorly characterized

Conflict of interest: The author has declared that no conflict of interest exists. Reference information: J Clin Invest. 2016;126(4):1216-1223. doi:10.1172/JCI81136. vesicular components that differ in size, including apoptotic bodies (1,000-5,000 nm), intermediate-sized microvesicles (200-1,000 $\mathrm{nm}$ ), and exosomes (30-150 nm). Exosomes, including TEX, are heterogeneous in size and functions but differ from other EVs because of their distinct biogenesis, which involves the endosomal compartment and is characteristic of all exosomes $(13,14)$. The molecular cargo exosomes carry is partly derived from the surface of parent tumor cells and from endosomes (14). This unique molecular signature discriminates among TEX produced by different tumor cells and distinguishes TEX from exosomes derived from normal cells (15).

Exosomes can only be visualized by electron microscopy (EM). Morphologically, TEX resemble other exosomes: they are spherical, membrane-bound vesicles that often measure less than $50 \mathrm{~nm}$ in diameter and form aggregates of various sizes. Preparation of TEX for EM may result in artifacts that are doughnut-shaped in appearance or smaller than expected in size as a result of shrinking. The EM of Epon-embedded exosome sections provides a more realistic view of TEX, as illustrated in Figure 1. Immuno-EM has confirmed the presence of FasL on the TEX surface (8), and by extension, it can be surmised that other immune-inhibitory molecules could be present on the TEX surface as well.

Western blots of TEX isolated from tumor cell supernatants and exosome fractions obtained from cancer patients' plasma confirm the expression of various immunosuppressive molecules, including death receptor ligands such as FasL or TRAIL, checkpoint receptor ligands such as PD-L1, inhibitory cytokines such as IL-10 and TGF- $\beta 1$, as well as prostaglandin $\mathrm{E}_{2}\left(\mathrm{PGE}_{2}\right)$ and ectoenzymes engaged in the adenosine pathway (CD39 and D73) (Figure 2). These soluble factors are known to be involved in tumor immune escape $(6,7,12)$. Soluble factors such as cytokines or cytokine receptors, which encounter each other in the endoplasmic reticulum, could be embedded in the exosome membrane and transported to the cell surface of parent cells. It is possible that exosomes carry and deliver cytokines to recipient cells in trans or cis configurations, thus expanding and magnifying the range of biological effects, including immune suppression, that these cytokinecytokine receptor complexes mediate. In addition to immunosup- 


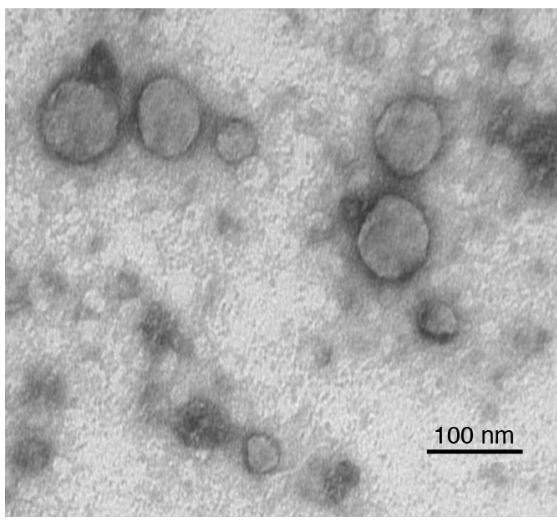

Figure 1. Electron micrograph of TEX produced by a human head and neck cancer cell line, PCI-13. TEX were isolated from the cell supernatant by ultracentrifugation. The pelleted TEX were fixed with glutaraldehyde, dehydrated, and embedded in Epon. Ultrathin sections were cut, stained with uranyl acetate, and examined by transmission EM. Note the variation in vesicle sizes. Image courtesy of S. Watkins (University of Pittsburgh, Pittsburgh, Pennsylvania, USA).

pressive cargo, TEX also carry tumor-associated antigens (TAAs), costimulatory molecules, and the MHC components, which enable them to stimulate immune cells (Figure 2). The phenotypic profile of TEX endows them with the ability to mediate either immune suppression or immune stimulation, presumably depending on the environment into which they are released by parent cells.

Because TEX can mediate either a loss or gain of antitumor immune responses $(16,17)$, a controversy has evolved concerning their biological role. Despite emerging evidence that the immunostimulatory or immunoinhibitory functions of TEX depend on the type of cargo and the functional status of immune cells in the tumor microenvironment (TME) and thus might be highly variable, it has been difficult to reconcile these two aspects of TEX functionality. Interestingly, in fields other than cancer, including inflammation, autoimmunity, transplantation, and pregnancy, the immunosuppressive potential of exosomes is not only acknowledged, but is explored for the development of novel therapeutic strategies (18-22). Given the immunoinhibitory nature of the TME in humans, it is unlikely that TEX, which increase in numbers with disease stage and progression $(1,8)$, are involved in boosting immune responses. In the TME, TEX are considered promoters of tumor-mediated immunosuppression. It has been suggested, however, that the immunopotentiating role of TEX could be harnessed for use in vaccination strategies, as TEX carry membrane-associated TAAs and could serve as components of antitumor vaccines (23).

\section{Isolation of TEX from body fluids of cancer patients}

Most of the studies performed to date used TEX isolated from supernatants of cultured tumor cells. In these supernatants, tumor cells are the only source of exosomes. To study TEX present in patients' body fluids, it is necessary to separate TEX from larger EVs and also from exosomes derived from nonmalignant cells. This requires the development of methods for the capture of TEX and their quantitative recovery. Fortunately, TEX carry membrane-embedded molecules that mimic those in parent tumor cells (24). Hence, Abs recognizing TAAs can be coated on beads and used for TEX capture (25). Immunocapture of TEX from plasma of acute myeloid leukemia (AML) patients with $\mathrm{CD} 34^{+}$blasts has been successful in our hands. The captured blast-derived exosomes were immunosuppressive, as measured by their ability to downregulate expression of the NK cell-activating receptor NKG2D in activated, normal NK cells (26). Others have used immune capture on beads to isolate glypican $1^{+}$ $\left(\mathrm{GPC1}^{+}\right)$exosomes from plasma of patients with early pancreatic cancer (27) or prostate-specific membrane antigen-carrying exosomes captured from peripheral blood of patients with prostate cancer (28). Methods for immunocapture of TEX from plasma of patients with other solid tumors are being developed. If successful, this strategy will make it possible to study TEX in parallel with exosomes produced by nonmalignant cells and determine which of the two fractions alters immune cells functions.

\section{TEX carry cargos derived from parent tumor cells}

TEX acquire their cargo from the parent tumor cell via the complex process of biogenesis (14). TEX originate from late endosomes and multivesicular bodies (MVBs) through a coordinated series of inward membrane invaginations $(14,29)$. Intraluminal vesicles formed in MVBs contain receptors and transmembrane proteins derived from the parent cell surface membrane as well as the cytosol. These parent cell components are sorted and packaged into TEX by the exosomal sorting complex responsible for transport (ESCRT) (14). It has been suggested that the sorting process may be parent cell specific, targeting sorted materials to a specific "address." When MVBs enclosing pools of future exosomes fuse with the cell membrane, TEX are released into the extracellular space, carrying information from the parent tumor cell to recipient

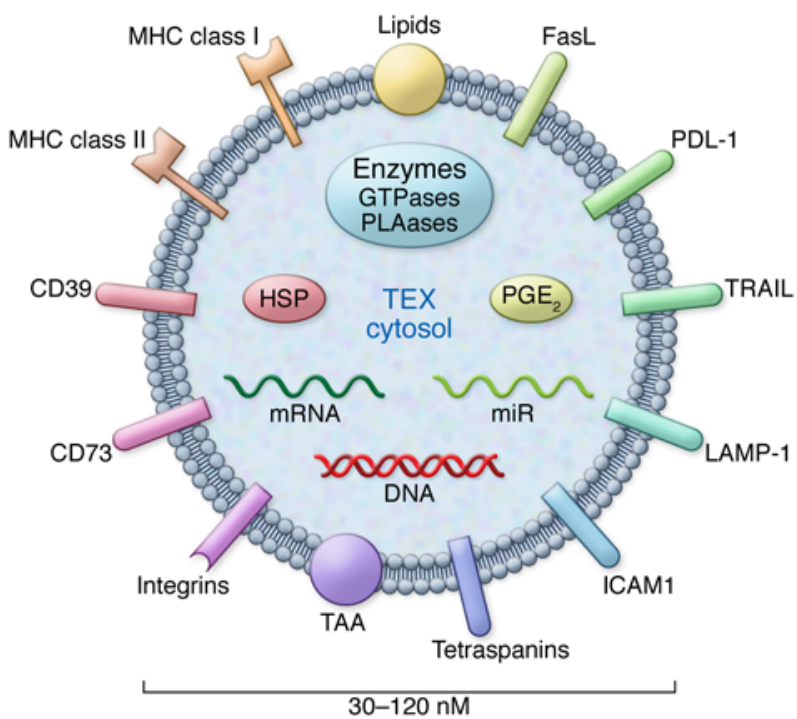

Figure 2. Components of the TEX cargo. TEX may contain immunoinhibitory ligands and immunostimulatory molecules. The intravesicular content includes nucleic acids and various cytosolic components from the cytosol of a parent cell. Reproduced with permission from Biochemical Society Transactions (50). 


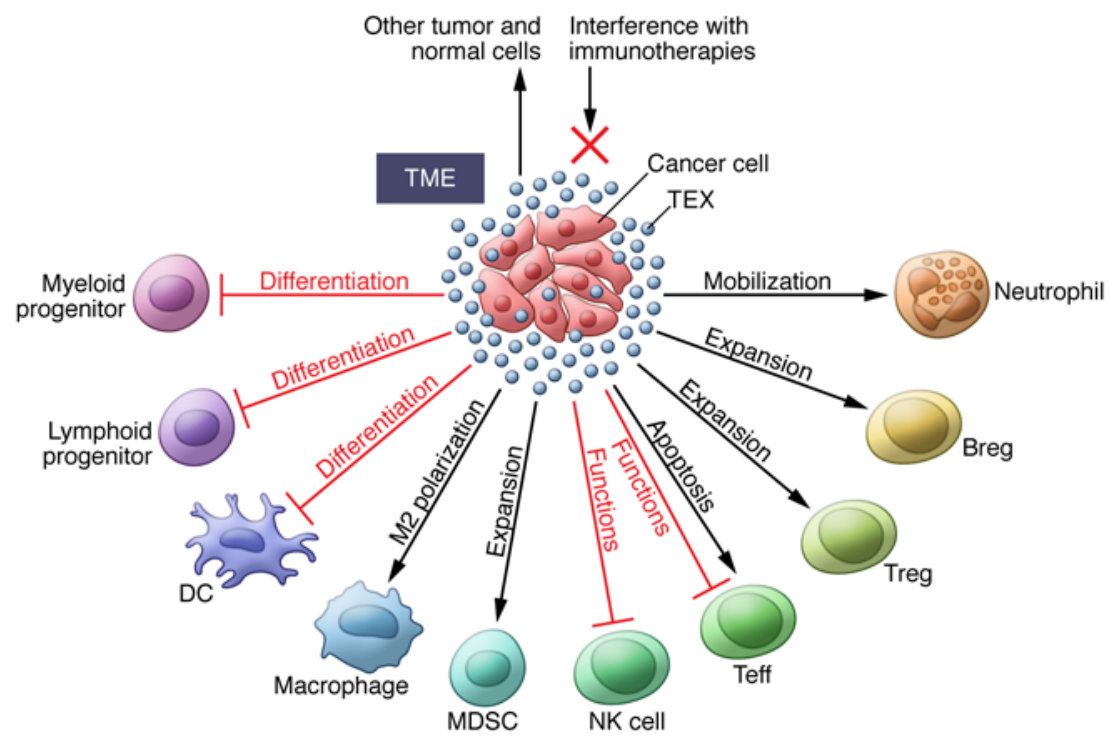

Figure 3. Effects of TEX on immune cells or immune cell progenitors. In the TME, TEX (in blue), produced and released by tumor cells, induce: (a) inhibition of functions necessary for antitumor responses; (b) apoptosis of activated Teffs; (c) expansion and upregulation of suppressive activity in Tregs, MDSCs, and regulatory B cells (Bregs); (d)interference with cellular differentiation; (e) polarization to tumor-promoting phenotypes; and (d) mobilization of cells to tumor. TEX carrying TAAs can interfere with immunotherapies. TEX also mediate autocrine effects and influence the functions of normal cells present in the TME. cells (29). The cargo delivery leads to notable biological effects in recipient cells, including changes in the cellular transcriptome and proteome as well as cellular functions $(30,31)$.

\section{Functional attributes of TEX}

Similar to other exosomes, TEX are involved in a broad variety of cellular functions and participate in physiological as well as pathological events (32). Foremost among TEX functions is information transfer from tumor cells to other malignant or normal cells $(7,33)$. TEX are well equipped to serve as communication vehicles. Their surface is decorated by the parent cell-derived signaling molecules, and their intravesicular content includes DNA, mRNA, and microRNA (miR) as well as enzymes and soluble factors, which are biologically active and capable of executing functional responses in target cells (34). Cancer cells secrete millions of TEX that are freely distributed throughout all body fluids, creating a communication network. Exosome levels in plasma and other body fluids of patients with cancer are frequently elevated (35). It has been suggested that stress, including hypoxia in the TME, accounts for this copious TEX secretion by tumor cells (36). TEX production and release by tumor cells was also reported to be regulated by p53 (37).

The communication network is entirely tumor driven and designed to promote tumor progression and metastasis, in part by silencing antitumor immune responses. The ability of TEX produced by mouse melanoma cells to educate and transform the bone marrow (BM) environment into a melanoma-promoting milieu has been elegantly demonstrated by Peinado and colleagues (38). TEX-mediated alterations of the BM are known to interfere with hematopoietic cell development and differentiation $(7,39)$. TEX have also been shown to interfere with functions of mature hematopoietic cells in the TME (7). TEX can induce immune suppression directly by delivering suppressive or apoptosis-inducing signals to activated immune cells (40), or indirectly by inducing the differentiation of Tregs and myeloid-derived suppressor cells (MDSCs) and supporting their expansion $(41,42)$. Direct effects of TEX on human T and NK cells have been examined by coincubation of these cells with TEX and subsequent assessments of
TEX-induced changes in responder cell function. Suppression of $\mathrm{T}$ cell functions was consistently recapitulated with exosomes or EVs isolated from patients' plasma, but not those isolated from normal donors' plasma (41). The delivery of TEX produced by murine tumor cell lines to tumor-bearing mice inhibited the frequency and cytolytic activity of NK cells, enhanced immunosuppressive activity of myeloid cells, and upregulated inflammatory cytokine production $(43,44)$. This in vivo modulation of immune cell functions by TEX was associated with tumor progression and metastasis formation. Exosomes made by DCs or B cells did not interfere with the functions of immune cells.

\section{Mechanisms of TEX-mediated immune suppression}

Clearly, the tumor-driven communication system is likely to be oriented toward effects and activities that benefit the tumor. To silence antitumor immune responses, the TEX cargo contains elements able to induce immune cell dysfunction in several different ways; however, the TEX must first interact with immune cells through one or more mechanisms (45). Ligands carried by TEX can be recognized by the cognate receptors on lymphocytes or antigens carried by TEX that bind to cellular MHC receptors. Through receptor-mediated uptake, TEX can directly fuse with the surface membrane and release their content into the cytoplasm. Phagocytic cells such as macrophages and DCs rapidly take up and internalize TEX. T cells do not seem to readily internalize TEX; instead, TEX interact with surface molecules to deliver signals that result in sustained $\mathrm{Ca}^{2+}$ flux and activation of downstream signaling molecules, leading to alterations in the recipient cell transcriptome (46). TEX-mediated signals can interfere with immune cell functions at multiple levels, and Figure 3 summarizes various cellular mechanisms responsible for exosome-mediated effects.

TEX deliver tolerogenic signals to immune cells. TEX carry inhibitory ligands that bind to cognate receptors on immune cells, inducing negative signaling (47). The two key receptors on immune cells, the T cell receptor (TCR) and the IL-2 receptor (IL-2R), are 
negatively regulated by TEX $(48,49)$. We have reported that TEX mediated dose- and time-dependent inhibition of $\mathrm{CD} 3 \zeta$ chain expression and reduced levels of mRNA coding for the CD3 $\zeta$ chain (50). It has been suggested, but not proven, that TEX, which carry MHC-peptide complexes as well as the immunosuppressive cargo, may preferentially inhibit tumor-specific T cells (41). Cognate interactions of MHC-peptide complexes carried by TEX with a TCR that is unable to signal via the $\zeta$ chain are likely to result in an abortive immune response. Even if these interactions lead to $\mathrm{T}$ cell activation, the absence of signals 2 and 3 (a costimulatory signal and cytokine stimulation, respectively) would inhibit $\mathrm{T}$ cell proliferation. We showed that TEX reduced JAK expression in activated $\mathrm{T}$ cells $(41,50)$. The integrity of the JAK pathway is critical for the functions of cytokines sharing the $\gamma$-chain of the IL-2R (IL-2, IL-7, IL-15); thus, suppression of IL-2R $\gamma$-chain phosphorylation levels leads to the failure of $\mathrm{T}$ cells to produce these cytokines and to proliferate. TEX also induced phosphorylation of STAT5 in activated $\mathrm{CD}^{+} \mathrm{T}$ cells and upregulated STAT5 phosphorylation in $\mathrm{CD}^{+} \mathrm{T}$ cells (50). Clayton and colleagues reported that TEX selectively impaired human lymphocyte responses to IL-2 (48). TEX-delivered signals trigger the activation of $\mathrm{NF}-\kappa \mathrm{B}$ and STAT3 (51) and alter cytokine profiles in T cells, reprogramming them toward the Th2 phenotype (51). TEX signal to monocytes, inducing secretion of the proinflammatory cytokines IL-6, TNF- $\alpha$, IL-1 $\beta$, and granulocyte-CSF (G-CSF) (52). Adenosine is a wellknown immunosuppressive factor that signals to effector $\mathrm{T}$ cells (Teffs) via the adenosine $A_{2 A}$ receptor (A2AR) to upregulate cAMP levels and inhibit Teff function (53). TEX carry CD39 and CD73, the ectonucleotidases responsible for ATP-dependent adenosine production, thereby serving as vehicles for the delivery of these enzymes to target cells (54). CD39+ Tregs in the TME are beneficiaries of this process, as continuous TEX-mediated CD73 delivery enables them to increase adenosine production and upregulate immunosuppressive functions (55). Emerging data implicate TEX in interference with other molecular pathways in immune cells (56). Given that TEX are ubiquitous in the TME, delivery of tolerogenic signals to the infiltrating immune cells appears to be one of their main functions.

TEX inhibit immune cell proliferation. Our ex vivo experiments showed that TEX inhibited the proliferation of human $\mathrm{CD} 8^{+} \mathrm{T}$ cells, but promoted that of $\mathrm{CD} 4^{+} \mathrm{T}$ cells. In contrast, control exosomes made by normal cells readily induced the proliferation of all $\mathrm{T}$ cells $(41,50)$. Further, TEX preferentially inhibited the proliferation of human melanoma-specific $\mathrm{CD}^{+} \mathrm{T}$ cells generated in cultures of human T cells with melanoma peptide-pulsed DCs (41). In vivo studies in mice also provided evidence that the transfer of exosomes from tumor-bearing mice to animals immunized with ovalbumin reduced the frequency and activity of antigen-specific T cells (57). These data suggest that TEX can inhibit antigen-specific $\mathrm{T}$ cell responses.

TEX induce apoptosis of activated $C D 8^{+}$Teffs. Nearly all CD $8^{+} \mathrm{T}$ lymphocytes in the circulation of cancer patients express surface CD95 (58), and many express programmed death 1 (PD-1) (59). Therefore, they are susceptible to apoptosis by TEX carrying the membrane form of FasL $(8,40)$ or programmed death ligand 1 (PD-L1), respectively. Expression levels of these apoptosis-inducing molecules in TEX were correlated with the frequency of apop- tosis-sensitive activated $\mathrm{CD}^{+} \mathrm{T}$ cells in the circulation of cancer patients. Importantly, there was a significant correlation between spontaneous apoptosis of circulating $\mathrm{CD}^{+} \mathrm{T}$ cells and the disease stage and prognosis $(8,58)$. TEX-mediated signals leading to apoptosis of activated $\mathrm{CD} 8^{+} \mathrm{T}$ cells were associated with early membrane changes (i.e., annexin $\mathrm{V}$ binding) in target cells, caspase 3 cleavage, cytochrome $\mathrm{C}$ release from mitochondria, loss of the mitochondrial membrane potential (MMP), and, finally, DNA fragmentation (60). The PI3K/AKT pathway is a key target of TEX in activated $\mathrm{CD}^{+} \mathrm{T}$ cells $(60,61)$. Recently, phosphatase and tensin homolog (PTEN), which regulates PI3K/AKT signaling, was found to be a component of the TEX cargo and to mediate phosphatase activity in recipient cells (62). Coincubation of activated $\mathrm{CD}^{+} \mathrm{T}$ cells with TEX caused dramatic, time-dependent AKT dephosphorylation and a concomitant downregulation of the expression levels of the antiapoptotic proteins BCL-2, BCL-xL, and MCl-1. At the same time, the proapoptotic protein Bax was upregulated by TEX $(49,60)$. Thus, TEX induce apoptosis of activated CD8 ${ }^{+} \mathrm{T}$ cells by engaging extrinsic and intrinsic apoptosis pathways (60). The in vitro data discussed above are consistent with reports of similar changes in the expression of the pro- or antiapoptotic family members in circulating $\mathrm{T}$ cells of patients with cancer $(61,63)$.

TEX suppress NK cell activity. The frequency and activity of NK cells are often depressed in cancer patients compared with age-matched, healthy individuals (64). Additionally, expression levels of the NK cell-activating receptors NKp30, NKp46, NKG2C, and NKG2D are low in cancer patients (35). TEX downregulate expression of NKG2D and reduce NK cell cytotoxicity $(35,64,65)$. At the time of diagnosis, TEX isolated from the plasma of AML patients showed MHC class I polypeptide-related sequences A and B (MICA and MICB), inhibited NK cell cytotoxicity, and depressed NKG2D expression in normal NK cells (35). The inhibitory effects of TEX were attributed to the presence of TGF- $\beta 1$, a cytokine known to suppress NK cell cytotoxicity. Inhibition of TGF- $\beta 1$ with neutralizing Abs partially abrogated TEXmediated suppression of NK cell activity (35). Our more recent data confirm that TEX from AML patients' plasma carry proTGF- $\beta 1$, latency-associated peptide (LAP), and mature TGF- $\beta 1$ in varying proportions and that TEX-mediated downregulation of NKG2D expression in activated NK cells is dependent on levels of mature TGF- $\beta 1$ carried by TEX (66).

TEX interfere with monocyte differentiation. Rivoltini and colleagues were the first to report that TEX inhibited human monocyte differentiation (67). Coincubation of peripheral blood monocytes (PBMCs) with TEX promoted their differentiation into TGF- $\beta$-expressing DCs, which also secreted $\mathrm{PGE}_{2}$ and interfered with cytotoxic T lymphocyte (CTL) generation. DCs generated in the presence of TEX expressed low levels of costimulatory molecules and induced dose-dependent inhibition of $\mathrm{T}$ cell proliferation. The results of these in vitro studies were later confirmed by in vivo experiments in mice (68).

TEX skew the differentiation of myeloid precursor cells into MDSCs. The in vivo experiments performed by Zhang and colleagues showed that TEX can channel myeloid precursor cells toward differentiation into MDSCs, which accumulate in murine tumor tissues, lymphoid organs, and blood (42). TEX-induced MDSC expansion was dependent on MyD88 signaling 
and required the presence of TGF- $\beta$ and $\mathrm{PGE}_{2}$ in the TEX cargo (69). MDSC accumulation has a two-fold effect on the immune response: first, with the paucity or absence of DCs, antigen processing and presentation are negatively affected, and, second, the newly minted MDSCs produce numerous immunosuppressive inhibitory factors, including NO and ROS, which cause nitration of TCRs or T cell apoptosis (70). Further, MDSCs consume arginine and cysteine, which are required for T cell activities (70). TEX isolated from body fluids of cancer patients converted the cytokine profile of a human monocytic cell line (THP-1) to an intensely proinflammatory type that would likely drive differentiation toward the MDSC phenotype (71).

TEX drive differentiation and expansion of Tregs. The frequency of circulating $\mathrm{CD}^{+} \mathrm{CD} 25^{\mathrm{hi}} \mathrm{FOXP}^{+}$Tregs is often elevated in patients with cancer (72). TEX induced the conversion of human conventional $\mathrm{CD}^{+}{ }^{+} \mathrm{CD} 25^{-} \mathrm{T}$ cells to $\mathrm{CD} 4^{+} \mathrm{CD} 25^{\mathrm{hi}} \mathrm{FOXP}^{+}$Tregs (41) in a TGF- $\beta 1$-dependent manner, increasing levels of phosphorylated SMAD2/3 and phosphorylated STAT3 (55), and promoted Treg proliferation in culture (55). TEX coincubated with neutralizing Abs against TGF- $\beta 1$ or IL-10 lost the ability to expand Tregs. In our hands, Tregs coincubated with TEX upregulated the expression levels of FasL, TGF- $\beta$, IL-10, CTL antigen 4 (CTLA4), granzyme $\mathrm{B}(\mathrm{GrB})$, and perforin and exhibited enhanced suppressor functions (55). Further, Tregs that proliferated in response to TEX were completely resistant to TEX-mediated apoptosis (55). Similar Tregenhancing effects of TEX were recently reported by others (73).

\section{TEX interfere with cancer immunotherapies}

As TEX are known to carry TAAs, they can efficiently bind and sequester tumor-reactive Abs and dramatically reduce binding of these Abs to tumor cells. This has been shown for trastuzumab in breast cancer therapy (74). HER2 ${ }^{+}$exosomes isolated from plasma of patients with breast cancer bound trastuzumab. Further, HER2 ${ }^{+}$ exosomes inhibited trastuzumab-mediated effects on the proliferation of SKBR3 cells, which overexpress HER2 (74). Ab sequestration also reduces $\mathrm{Ab}$-dependent cell-mediated cytotoxicity (ADCC) by immune effector cells, one of the major mechanisms of therapeutic activity of anticancer Abs (75). In a model of an aggressive B cell lymphoma, TEX were shown to bind and consume complement, thereby protecting tumor cells from complement-dependent cytolysis (70). It can also be surmised that $\mathrm{TAA}^{+}$ TEX could interfere with antigen-specific recognition of tumor cells by antitumor-reactive CTLs generated as a result of vaccination therapies or adoptive transfer of immune cells to patients with cancer. In aggregate, the available insights into the molecular cargo of TEX suggest that TEX are likely to play an important role in modulating the sensitivity of tumor cells to immune therapies and in antitumor activities of immune effector cells.

\section{Molecular and genetic profiles of TEX}

Attempts to link the immunosuppressive effects of TEX to their molecular and genetic profiles as well as extensive studies of the TEX proteome and transcriptome are in progress.

Protein content of TEX. Protein levels of exosome fractions in the plasma of patients with different malignancies were reported to correlate with disease activity, tumor grade, tumor stage, response to therapy, and survival $(38,70,76)$. For example, in patients with recurrent malignant glioma who participated in a phase I/II vaccination trial, protein levels of plasma exosome fractions obtained at diagnosis and prior to vaccination were elevated and correlated with the WHO tumor grade (77). Protein levels in plasma exosome fractions decreased rapidly after vaccination, but only in patients who had evidence of immunological and clinical response to the vaccine, suggesting that the recovery of immune responses after the vaccine was related to a decrease in the number or functions of potentially immunosuppressive exosomes.

The composition of immunosuppressive factors, such as membrane-associated TGF- $\beta 1$, in the exosome cargo was found to change with therapy. Alterations in levels of the TGF- $\beta 1$ propeptide LAP and the mature form of TGF- $\beta 1$ in exosomes isolated from AML patients' plasma correlated with patients' responses to chemotherapy (65). The data suggesting that total or individual protein levels in TEX might correlate with cancer progression or responses to therapy have led to extensive proteomic analyses of EVs isolated from tumor cell supernatants and to the identification of several thousand different molecules carried by EVs and listed in the Vesiclepedia (formerly ExoCarta) databases $(78,79)$. These data do not distinguish between TEX and larger EVs, but they indicate that the protein signatures of EVs produced by different types of tumor cells are distinct (implying cancer cell-type specificity) and differ from the signatures of EVs produced by nonmalignant cells (80). The detection of immune inhibitory cytokines and ligands by liquid chromatography-tandem mass spectroscopy (LC-MS/MS) in EVs from patients' plasma has been less successful and seems to require highly sensitive techniques, largely due to contaminating plasma proteins, which mask genuine TEX-associated components. So far, Western blots, which allow for Ab-based detection of inhibitory proteins, provide the only solid link between their expression in the TEX cargo and immune inhibitory activity measured in vitro.

Nucleic acid content of TEX. The presence in the TEX cargo of DNA, mRNA, and miRs is important for the role of TEX as information-carrying vehicles. TEX derived from tumor cell lines and EVs from the plasma of cancer patients contain double-stranded genomic DNA (gDNA) (81). Analyses of gDNA fragments of MLH1, PTEN, or TP53 genes showed that different exosomes had distinct gDNA content that could include specific mutations $(81,82)$. TEX have the ability to carry and transfer oncogenic mutations to recipient cells (83).

TEX were reported to contain about 10,000 distinct mRNA species, many of which are known to be involved in critical cellular activities, including immune regulation and inflammation (31). In our hands, TEX isolated from the plasma of 20 patients with recurrent glioma participating in a clinical vaccination trial (77) yielded sufficient quantities of mRNA for quantitative reverse transcription PCR analyses. Expression levels of 24 immune-regulatory genes were measured in TEX recovered from the paired pre- and post-vaccination plasma samples (77). Expression levels of 4 of the 24 genes (IL8, TGFB, TIMP1, and ZAP7O) were significantly decreased in exosomes recovered after vaccination. These four genes are known to be related to angiogenesis, immune regulation, and clinical outcome in glioma. Importantly, these vaccine-induced changes in the mRNA transcripts occurred only in patients who exhibited immunological and clinical responses to the vaccine, as three of four immunologic responders were alive 64 
months after vaccination (77). This small retrospective vaccination study of patients with advanced disease showed that measurements of changes in expression levels of immune-related genes in exosomes were useful in identifying vaccine-responsive patients. As total exosomes recovered from plasma and not isolated TEX were evaluated, it is likely that the transcriptional changes we observed occurred in immune cell-derived exosomes rather than in TEX. The study results suggest that analyses of mRNA in plasma exosomes of cancer patients treated with immune therapies might provide useful clinical and prognostic information.

TEX cargo is rich in miRs (84). TEX have been called "oncomirs," and the miR content of TEX has been extensively investigated (85). miRs regulate gene expression in recipient cells by either repressing translation or inducing degradation of multiple target mRNAs, depending on the cellular context $(84,86)$. The transfer of miRs from tumor to immune cells alters their functions, usually downregulating antitumor activities and promoting tumorigenesis (84). Tumor-associated miRs, such as miR-21, miR-155, miR-146a, and miR-568, which have been frequently identified as components of the TEX cargo, regulate the differentiation and functions of various immune cells, often inhibiting effector functions or inducing apoptosis (87-91). Exosomes in the plasma of patients with different cancer types carry distinct, cancer-specific miR signatures, which appear to correlate with the cancer progression and responses to therapy $(92,93)$.

\section{TEX as cancer biomarkers}

The immunosuppressive profile of TEX in body fluids has the potential to serve as a readily accessible noninvasive measure of tumor-induced immune dysfunction in cancer. Recent data support the role of immune dysfunction in cancer progression and poor outcome (72, 94). By the same token, reversal of tumor-induced immune suppression by immune therapies such as immune checkpoint inhibitors are better predictors of outcome in many, although not all, cancer patients (95). In this context, TEX, and possibly immune cell-derived exosomes, could serve as surrogate markers of immune dysfunction or immune recovery and, by extension, of poor or good disease outcome. Further, by using TEX as tumor cell surrogates and exosomes derived from $\mathrm{TCR}^{+}$or $\mathrm{CD}^{+} \mathrm{T}$ cell-derived exosomes as antitumor immune response surrogates, it might be possible to develop two biomarkers of cancer progression or response to therapy. The potential of TEX for noninvasive cancer monitoring has been recently reviewed (96), and the use of TEX as biomarkers awaits further studies and validation in prospective clinical protocols.

\section{Conclusions}

TEX are rapidly emerging as a critical component of a tumororchestrated information system that is designed to facilitate tumor immune escape and promote tumor growth. TEX carrying immunosuppressive cargos deliver molecular signals to immune cells, which alter the functions of these cells, and nucleic acids, which can reprogram their genetic code. The ubiquitous presence of TEX in body fluids of cancer patients explains the various defects observed in immune cells of these patients. TEX-mediated effects may be responsible for the lack of response to cancer immunotherapies. TEX used as biomarkers could potentially serve as a noninvasive strategy to monitor tumor progression or response to therapy.

\section{Acknowledgments}

This work was supported in part by NIH grant R01 CA16862 (to T.L. Whiteside).

Address correspondence to: Theresa L. Whiteside, University of Pittsburgh, Hillman Cancer Center, 5117 Centre Avenue, Suite 1.27, Pittsburgh, Pennsylvania 15213, USA. Phone: 412.624.0096; E-mail: whitesidetl@upmc.edu.
1. Keller S, Ridinger J, Rupp AK, Janssen JW, Altevogt P. Body fluid derived exosomes as a novel template for clinical diagnostics. J Transl Med. 2011;9:86.

2. Poliakov A, Spilman M, Dokland T, Amling CL, Mobley JA. Structural heterogeneity and protein composition of exosome-like vesicles (prostasomes) in human semen. Prostate. 2009;69(2):159-167.

3. Palanisamy V, Sharma S, Deshpande A, Zhou H, Gimzewski J, Wong DT. Nanostructural and transcriptomic analyses of human saliva derived exosomes. PLoS One. 2010;5(1):e8577.

4. Skriner K, Adolph K, Jungblut PR, Burmester GR. Association of citrullinated proteins with synovial exosomes. Arthritis Rheum. 2006;54(12):3809-3814.

5. Zhang HG, Grizzle WE. Exosomes: a novel pathway of local and distant intercellular communication that facilitates the growth and metastasis of neoplastic lesions. Am J Pathol. 2014;184(1):28-41.

6. Bobrie A, Thery C. Exosomes and communication between tumours and the immune system: are all exosomes equal? Biochem Soc Trans. 2013;41(1):263-267.
7. Boyiadzis M, Whiteside TL. Information transfer by exosomes: A new frontier in hematologic malignancies. Blood Rev. 2015;29(5):281-290.

8. Kim JW, Wieckowski E, Taylor DD, Reichert TE, Watkins S, Whiteside TL. Fas ligand-positive membranous vesicles isolated from sera of patients with oral cancer induce apoptosis of activated T lymphocytes. Clin Cancer Res. 2005;11(3):1010-1020.

9. Pan BT, Johnstone RM. Fate of the transferrin receptor during maturation of sheep reticulocytes in vitro: selective externalization of the receptor. Cell. 1983;33(3):967-978.

10. Raposo G, Stoorvogel W. Extracellular vesicles: exosomes, microvesicles, and friends. JCell Biol. 2013;200(4):373-383.

11. Greening DW, Gopal SK, Xu R, Simpson RJ, Chen $\mathrm{W}$. Exosomes and their roles in immune regulation and cancer. Semin Cell Dev Biol. 2015;40:72-81.

12. Whiteside TL. The tumor microenvironment and its role in promoting tumor growth. Oncogene. 2008;27(45):5904-5912.

13. Colombo M, et al. Analysis of ESCRT functions in exosome biogenesis, composition and secretion highlights the heterogeneity of extracellular vesicles. J Cell Sci. 2013;126(pt 24):5553-5565.
14. Cocucci E, Meldolesi J. Ectosomes and exosomes: shedding the confusion between extracellular vesicles. Trends Cell Biol. 2015;25(6):364-372.

15. Wieckowski E, Whiteside TL. Human tumorderived vs dendritic cell-derived exosomes have distinct biologic roles and molecular profiles. Immunol Res. 2006;36(1-3):247-254.

16. Robbins PD, Morelli AE. Regulation of immune responses by extracellular vesicles. Nat Rev Immunol. 2014;14(3):195-208.

17. Gabrielsson S, Scheynius A. Exosomes in immunity and cancer - friends or foes? Semin Cancer Biol. 2014;28:1-2.

18. Stenqvist AC, Nagaeva O, Baranov V, Mincheva-Nilsson L. Exosomes secreted by human placenta carry functional Fas ligand and TRAIL molecules and convey apoptosis in activated immune cells, suggesting exosome-mediated immune privilege of the fetus. JImmunol. 2013;191(11):5515-5523.

19. Sadallah S, Eken C, Schifferli JA. Ectosomes as modulators of inflammation and immunity. Clin Exp Immunol. 2011;163(1):26-32.

20. Agarwal A, et al. Regulatory T cell-derived exosomes: possible therapeutic and diagnostic tools in transplantation. Front Immunol. 2014;5:555. 
21. Yu L, et al. Exosomes with membrane-associated TGF-beta1 from gene-modified dendritic cells inhibit murine EAE independently of $\mathrm{MHC}$ restriction. Eur JImmunol. 2013;43(9):2461-2472.

22. Kim SH, et al. Exosomes derived from genetically modified DC expressing FasL are anti-inflammatory and immunosuppressive. Mol Ther. 2006;13(2):289-300.

23. Gu X, Erb U, Buchler MW, Zoller M. Improved vaccine efficacy of tumor exosome compared to tumor lysate loaded dendritic cells in mice. Int J Cancer. 2015;136(4):E74-E84.

24. Iero M, et al. Tumour-released exosomes and their implications in cancer immunity. Cell Death Differ. 2008;15(1):80-88.

25. Taylor DD, Gercel-Taylor C. The origin, function, and diagnostic potential of RNA within extracellular vesicles present in human biological fluids. Front Genet. 2013;4:142.

26. Hong CS, Muller L, Boyiadzis M, Whiteside TL. Isolation and characterization of $\mathrm{CD} 34^{+}$blastderived exosomes in acute myeloid leukemia. PLoS One. 2014;9(8):e103310.

27. Melo SA, et al. Glypican-1 identifies cancer exosomes and detects early pancreatic cancer. Nature. 2015;523(7559):177-182.

28. Mizutani K, et al. Isolation of prostate cancer-related exosomes. Anticancer Res. 2014;34(7):3419-3423.

29. Brinton LT, Sloane HS, Kester M, Kelly KA. Formation and role of exosomes in cancer. Cell Mol Life Sci. 2015;72(4):659-671.

30. Vlassov AV, Magdaleno S, Setterquist R, Conrad R. Exosomes: current knowledge of their composition, biological functions, and diagnostic and therapeutic potentials. Biochim Biophys Acta. 2012;1820(7):940-948.

31. Skog J, et al. Glioblastoma microvesicles transport RNA and proteins that promote tumour growth and provide diagnostic biomarkers. Nat Cell Biol. 2008;10(12):1470-1476.

32. van der Pol E, Boing AN, Harrison P, Sturk A, Nieuwland R. Classification, functions, and clinical relevance of extracellular vesicles. Pharmacol Rev. 2012;64(3):676-705.

33. Martins VR, Dias MS, Hainaut P. Tumorcell-derived microvesicles as carriers of molecular information in cancer. Curr Opin Oncol. 2013;25(1):66-75.

34. Record M, Subra C, Silvente-Poirot S, Poirot M. Exosomes as intercellular signalosomes and pharmacological effectors. Biochem Pharmacol. 2011;81(10):1171-1182.

35. Szczepanski MJ, Szajnik M, Welsh A, Whiteside TL, Boyiadzis M. Blast-derived microvesicles in sera from patients with acute myeloid leukemia suppress natural killer cell function via membrane-associated transforming growth factor- $\beta 1$. Haematologica. 2011;96(9):1302-1309.

36. King HW, Michael MZ, Gleadle JM. Hypoxic enhancement of exosome release by breast cancer cells. BMC Cancer. 2012;12:421.

37. Abd Elmageed ZY, et al. Neoplastic reprogramming of patient-derived adipose stem cells by prostate cancer cell-associated exosomes. Stem Cells. 2014;32(4):983-997.

38. Peinado H, et al. Melanoma exosomes educate bone marrow progenitor cells toward a pro-met- astatic phenotype through MET. Nat Med. 2012;18(6):883-891.

39. Gross JC, Chaudhary V, Bartscherer K, Boutros M. Active Wnt proteins are secreted on exosomes. Nat Cell Biol. 2012;14(10):1036-1045.

40. Andreola G, et al. Induction of lymphocyte apoptosis by tumor cell secretion of FasL-bearing microvesicles. JExp Med. 2002;195(10):1303-1316.

41. Wieckowski EU, Visus C, Szajnik M, Szczepanski MJ, Storkus WJ, Whiteside TL. Tumor-derived microvesicles promote regulatory $\mathrm{T}$ cell expansion and induce apoptosis in tumor-reactive activated $\mathrm{CD}^{+} \mathrm{T}$ lymphocytes. J Immunol. 2009;183(6):3720-3730.

42. Xiang $X$, et al. Induction of myeloid-derived suppressor cells by tumor exosomes. Int J Cancer. 2009;124(11):2621-2633.

43. Liu C, et al. Murine mammary carcinoma exosomes promote tumor growth by suppression of NK cell function. JImmunol. 2006;176(3):1375-1385.

44. Pucci F, Pittet MJ. Molecular pathways: tumor-derived microvesicles and their interactions with immune cells in vivo. Clin Cancer Res. 2013;19(10):2598-2604.

45. Mulcahy LA, Pink RC, Carter DR. Routes and mechanisms of extracellular vesicle uptake. J Extracell Vesicles. 2014;3.

46. Zhang X, Yuan X, Shi H, Wu L, Qian H, Xu W. Exosomes in cancer: small particle, big player. J Hematol Oncol. 2015;8:83.

47. Gutierrez-Vazquez C, Villarroya-Beltri C, Mittelbrunn M, Sanchez-Madrid F. Transfer of extracellular vesicles during immune cell-cell interactions. Immunol Rev. 2013;251(1):125-142.

48. Clayton A, Mitchell JP, Court J, Mason MD, Tabi Z. Human tumor-derived exosomes selectively impair lymphocyte responses to interleukin-2. Cancer Res. 2007;67(15):7458-7466.

49. Taylor DD, Gercel-Taylor C, Lyons KS, Stanson J, Whiteside TL. T-cell apoptosis and suppression of T-cell receptor/CD3-zeta by Fas ligand-containing membrane vesicles shed from ovarian tumors. Clin Cancer Res. 2003;9(14):5113-5119.

50. Whiteside TL. Immune modulation of T-cell and NK (natural killer) cell activities by TEXs (tumour-derived exosomes). Biochem Soc Trans. 2013;41(1):245-251.

51. Bretz NP, et al. Body fluid exosomes promote secretion of inflammatory cytokines in monocytic cells via Toll-like receptor signaling. J Biol Chem. 2013;288(51):36691-36702.

52. Chalmin F, et al. Membrane-associated Hsp72 from tumor-derived exosomes mediates STAT3-dependent immunosuppressive function of mouse and human myeloid-derived suppressor cells. J Clin Invest. 2010;120(2):457-471.

53. Muller-Haegele S, Muller L, Whiteside TL. Immunoregulatory activity of adenosine and its role in human cancer progression. Expert Rev Clin Immunol. 2014;10(7):897-914.

54. Schuler PJ, et al. Human CD4(+) CD39(+) regulatory $\mathrm{T}$ cells produce adenosine upon co-expression of surface CD73 or contact with CD73(+) exosomes or CD73(+) cells. Clin Exp Immunol. 2014;177(2):531-543.

55. Szajnik M, Czystowska M, Szczepanski MJ, Mandapathil M, Whiteside TL. Tumor-derived microvesicles induce, expand and up-regulate biological activities of human regulatory $\mathrm{T}$ cells (Treg). PLoS One. 2010;5(7):e11469.

56. Luga V, et al. Exosomes mediate stromal mobilization of autocrine Wnt-PCP signaling in breast cancer cell migration. Cell. 2012;151(7):1542-1556.

57. Yang C, Kim SH, Bianco NR, Robbins PD. Tumor-derived exosomes confer antigen-specific immunosuppression in a murine delayed-type hypersensitivity model. PLoS One. 2011;6(8):e22517.

58. Hoffmann TK, et al. Spontaneous apoptosis of circulating $\mathrm{T}$ lymphocytes in patients with head and neck cancer and its clinical importance. Clin Cancer Res. 2002;8(8):2553-2562.

59. Schuler PJ, et al. Phenotypic and functional characteristics of $\mathrm{CD} 4^{+} \mathrm{CD}^{2} 9^{+} \mathrm{FOXP}^{+}$and $\mathrm{CD} 4{ }^{+}$ $\mathrm{CD}^{3}{ }^{+} \mathrm{FOXP}^{-} \mathrm{T}$-cell subsets in cancer patients. Eur J Immunol. 2012;42(7):1876-1885.

60. Czystowska M, et al. Mechanisms of T-cell protection from death by IRX-2: a new immunotherapeutic. Cancer Immunol Immunother 2011;60(4):495-506.

61. Czystowska M, et al. IRX-2, a novel immunotherapeutic, protects human $\mathrm{T}$ cells from tumor-induced cell death. Cell Death Differ. 2009;16(5):708-718.

62. Putz U, et al. The tumor suppressor PTEN is exported in exosomes and has phosphatase activity in recipient cells. Sci Signal. 2012;5(243):ra70.

63. Kim JW, Tsukishiro T, Johnson JT, Whiteside TL. Expression of pro- and antiapoptotic proteins in circulating $\mathrm{CD} 8^{+} \mathrm{T}$ cells of patients with squamous cell carcinoma of the head and neck. Clin Cancer Res. 2004;10(15):5101-5110.

64. Clayton A, Mitchell JP, Court J, Linnane S, Mason MD, Tabi Z. Human tumor-derived exosomes down-modulate NKG2D expression. J Immunol. 2008;180(11):7249-7258.

65. Mincheva-Nilsson L, Baranov V. Cancer exosomes and NKG2D receptor-ligand interactions: impairing NKG2D-mediated cytotoxicity and anti-tumour immune surveillance. Semin Cancer Biol. 2014;28:24-30.

66. Hong CS, Muller L, Whiteside TL, Boyiadzis M. Plasma exosomes as markers of therapeutic response in patients with acute myeloid leukemia. Front Immunol. 2014;5:160.

67. Valenti R, Huber V, Iero M, Filipazzi P, Parmiani G, Rivoltini L. Tumor-released microvesicles as vehicles of immunosuppression. Cancer Res. 2007;67(7):2912-2915

68. Yu S, et al. Tumor exosomes inhibit differentiation of bone marrow dendritic cells. JImmunol. 2007;178(11):6867-6875.

69. Liu Y, et al. Contribution of MyD88 to the tumor exosome-mediated induction of myeloid derived suppressor cells. Am J Pathol. 2010;176(5):2490-2499.

70. Filipazzi P, Burdek M, Villa A, Rivoltini L, Huber V. Recent advances on the role of tumor exosomes in immunosuppression and disease progression. Semin Cancer Biol. 2012;22(4):342-349.

71. Altevogt P, Bretz NP, Ridinger J, Utikal J, Umansky V. Novel insights into exosome-induced, tumor-associated inflammation and immunomodulation. Semin Cancer Biol. 2014;28:51-57.

72. Whiteside TL. Immune responses to cancer: are they potential biomarkers of prognosis? Front 
Oncol. 2013;3:107.

73. Mrizak D, Martin N, Barjon C, et al. Effect of nasopharyngeal carcinoma-derived exosomes on human regulatory T cells. J Natl Cancer Inst. 2015;107(1):363.

74. Ciravolo V, et al. Potential role of HER2-overexpressing exosomes in countering trastuzumab-based therapy. JCell Physiol. 2012;227(2):658-667.

75. Battke C, et al. Tumour exosomes inhibit binding of tumour-reactive antibodies to tumour cells and reduce ADCC. Cancer Immunol Immunother. 2011;60(5):639-648.

76. Bergmann C, et al. Tumor-derived microvesicles in sera of patients with head and neck cancer and their role in tumor progression. Head Neck. 2009;31(3):371-380.

77. Muller L, Muller-Haegele S, Mitsuhashi M, Gooding W, Okada H, Whiteside TL. Exosomes isolated from plasma of glioma patients enrolled in a vaccination trial reflect antitumor immune activity and might predict survival. Oncoimmunology. 2015;4(6):e1008347.

78. Kim DK, et al. EVpedia: an integrated database of high-throughput data for systemic analyses of extracellular vesicles. J Extracell Vesicles. 2013;2.

79. Kalra H, et al. Vesiclepedia: a compendium for extracellular vesicles with continuous community annotation. PLoS Biol. 2012;10(12):e1001450.

80. Amorim M, Fernandes G, Oliveira P, Martins-de-
Souza D, Dias-Neto E, Nunes D. The overexpression of a single oncogene (ERBB2/HER2) alters the proteomic landscape of extracellular vesicles. Proteomics. 2014;14(12):1472-1479.

81. Lazaro-Ibanez E, et al. Different gDNA content in the subpopulations of prostate cancer extracellular vesicles: apoptotic bodies, microvesicles, and exosomes. Prostate. 2014;74(14):1379-1390.

82. Kahlert C, et al. Identification of double-stranded genomic DNA spanning all chromosomes with mutated KRAS and p53 DNA in the serum exosomes of patients with pancreatic cancer. J Biol Chem. 2014;289(7):3869-3875.

83. Saleem SN, Abdel-Mageed AB. Tumor-derived exosomes in oncogenic reprogramming and cancer progression. Cell Mol Life Sci. 2015;72(1):1-10.

84. Dai R, Ahmed SA. MicroRNA, a new paradigm for understanding immunoregulation, inflammation, and autoimmune diseases. Transl Res. 2011;157(4):163-179.

85. Esquela-Kerscher A, Slack FJ. Oncomirs microRNAs with a role in cancer. Nat Rev Cancer. 2006;6(4):259-269.

86. Ye SB, et al. Tumor-derived exosomes promote tumor progression and $\mathrm{T}$-cell dysfunction through the regulation of enriched exosomal microRNAs in human nasopharyngeal carcinoma. Oncotarget. 2014;5(14):5439-5452.

87. Carissimi C, et al. miR-21 is a negative modulator of T-cell activation. Biochimie. 2014;107(pt B):319-326.
88. Curtale G, et al. An emerging player in the adaptive immune response: microRNA-146a is a modulator of IL-2 expression and activation-induced cell death in T lymphocytes. Blood. 2010;115(2):265-273.

89. Li W, et al. MiR-568 inhibits the activation and function of CD4(+) T cells and Treg cells by targeting NFAT5. Int Immunol. 2014;26(5):269-281.

90. Huan J, et al. RNA trafficking by acute myelogenous leukemia exosomes. Cancer Res. 2013;73(2):918-929.

91. Dunand-Sauthier I, et al. Silencing of c-Fos expression by microRNA- 155 is critical for dendritic cell maturation and function. Blood. 2011;117(17):4490-4500.

92. Brase JC, et al. Circulating miRNAs are correlated with tumor progression in prostate cancer. Int J Cancer. 2011;128(3):608-616.

93. Gracias DT, Katsikis PD. MicroRNAs: key components of immune regulation. Adv Exp Med Biol. 2011;780:15-26.

94. Baxevanis CN, Anastasopoulou EA, Voutsas IF, Papamichail M, Perez SA. Immune biomarkers: how well do they serve prognosis in human cancers? Expert Rev Mol Diagn. 2015;15(1):49-59.

95. Pardoll DM. The blockade of immune checkpoints in cancer immunotherapy. Nat Rev Cancer. 2012;12(4):252-264.

96. Whiteside TL. The potential of tumor-derived exosomes for noninvasive cancer monitoring. Expert Rev Mol Diagn. 2015;15(10):1293-1310. 